\title{
Laparoscopic nephrectomy for massive polycystic kidney disease: Updated technique and outcomes
}

\author{
Rahul Kumar Bansal, MBBS, MS, MCh; Anil Kapoor, MD, FRCSC \\ McMaster Institute of Urology, St. Joseph's Healthcare, Hamilton, ON
}

Cite as: Can Urol Assoc J 2014;8(9-10):341-5. http://dx.doi.org/10.5489/cuaj.2097

Published online October 13, 2014.

\section{Abstract}

Introduction: We present our technique of laparoscopic nephrectomy for massive polycystic kidneys in patients with autosomal dominant polycystic kidney disease (ADPKD) and review the outcome analysis of our experience.

Methods: We retrospectively reviewed all transperitoneal laparoscopic nephrectomies done for polycystic kidneys at a university hospital. Our technique included three 12-mm ports with additional one or two 5-mm ports, with usage of retraction devices, such as the Jarit PEER retractor (J. Jamner Surgical Instruments, Inc, Hawthorne, NY).

Results: In total, 39 (left 14, right 25) laparoscopic nephrectomies were performed in 32 patients (male 21, female 11). Surgical indications were varied: to create space for future renal transplant in 21 (54\%), to alleviate pain in $16(41 \%)$, to prevent recurrent urosepsis in $2(5 \%)$, to prevent recurrent bleeding which would require transfusions in 2 (5\%) and to remove a renal tumour in 1 kidney $(2.5 \%)$. Four patients had surgery for more than one reason. The mean age and body mass index were 52.2 years (range: $29-72$ ) and $26.9 \mathrm{~kg} / \mathrm{m}^{2}$ (range: $21.6-34.0$ ), respectively. The mean preoperative hemoglobin and serum creatinine levels were $131.6 \mathrm{~g} / \mathrm{L}$ (range: 107-171) and $514 \mu \mathrm{mol} / \mathrm{L}$ (range: 84-923), respectively; 26 (81\%) patients were on dialysis. The mean operative time and estimated blood loss were 185 minutes (range: 113-287) and $94 \mathrm{~mL}$ (range: 10-350), respectively. No patient required open conversion. The mean specimen size was $24.2 \mathrm{~cm}$ (range: 15-38); weight $1515 \mathrm{~g}$ (range: 412-4590) and the length of extraction incision was $9.2 \mathrm{~cm}$ (range: 6-13). There were 1 Grade 22 (2.5\%), 2 Grade 3b (5\%) and 1 Grade $4 a-d(2.5 \%)$ complications. The mean length of stay was 4.5 days (range: $3-8$ ).

Conclusions: Our technique of laparoscopic nephrectomy for massively enlarged polycystic kidneys in ADPKD is safe and offers all the advantages of minimal access surgery, such as smaller incision, decreased estimated blood loss, excellent cosmesis and faster recovery.

\section{Introduction}

Autosomal dominant polycystic kidney disease (ADPKD) represents one of the most common genetic disorders affecting kidneys (approximate incidence 1 in 500 to 1000), characterized by the presence of large number of fluid filled cysts in both kidneys. It affects people from all ethnic groups. ${ }^{1}$ Patients usually present around the fourth decade of life and develop end-stage renal disease after about 5 to 10 years of diagnosis of renal impairment. ${ }^{1}$ Renal manifestations include the presence and growth of multiple cysts, hypertension (50\%-100\%); pain (60\%) due to cyst rupture, hemorrhage, infection, stone or urinary tract infection and renal failure (50\%). Extrarenal manifestations are cysts in the liver, intracranial aneurysms, mitral valve prolapse and colonic diverticulosis. ${ }^{2}$

There is no consensus regarding appropriate treatment for symptomatic patients with ADPKD. Bilateral nephrectomy adequately treats symptoms, but initial studies report high complication rates, with $38 \%$ morbidity and $3 \%$ mortality. ${ }^{3}$ Concerns regarding the graft damage and sequelae of anephric state decrease the nephrectomy rate for ADPKD, ${ }^{4}$ but Sulikowski and colleagues reported that more than $40 \%$ of patients without pretransplant nephrectomy required it later because of various complications in native polycystic kidneys. ${ }^{5}$ Since the first description of laparoscopic nephrectomy for polycystic kidney in ADPKD by Elashry and colleagues, which decreased the morbidity of open nephrectomy, ${ }^{6}$ we and several other authors have reported their experience of laparoscopic nephrectomy for unilateral or bilateral polycystic kidneys. ${ }^{7-9}$

We hereby describe our updated technique and outcome of laparoscopic nephrectomy for massive polycystic kidneys.

\section{Methods}

After receiving ethics review board approval, retrospective review was done for all laparoscopic nephrectomies 
performed for polycystic kidneys in patients with ADPKD. These procedures were performed over a 7-year period from April 2004 to January 2011. Data were analyzed and surgical outcomes were reported. Demographics were noted and preoperative and perioperative data were collected. Complications were reported according to the modified Clavien Classification for surgical complications. ${ }^{10}$

Patients were followed up for a median period of 18 months postoperatively. One principal surgeon performed laparoscopic nephrectomies (AK) with assistance from residents and fellows.

\section{Laparoscopic nephrectomy for ADPKD}

After appropriate imaging was done to visualize renal anatomy (Fig.1, part A), informed consent was obtained from all patients for laparoscopic nephrectomy, and a preoperative antibiotic was administered routinely. Bowel preparation was not routinely performed for upper urinary tract procedures.

Our technique, with some modifications, of laparoscopic nephrectomy for patients with ADPKD has been described. ${ }^{8,9}$ The patient is placed in the lateral decubitus position and the operative table is flexed to open the costophrenic angle.

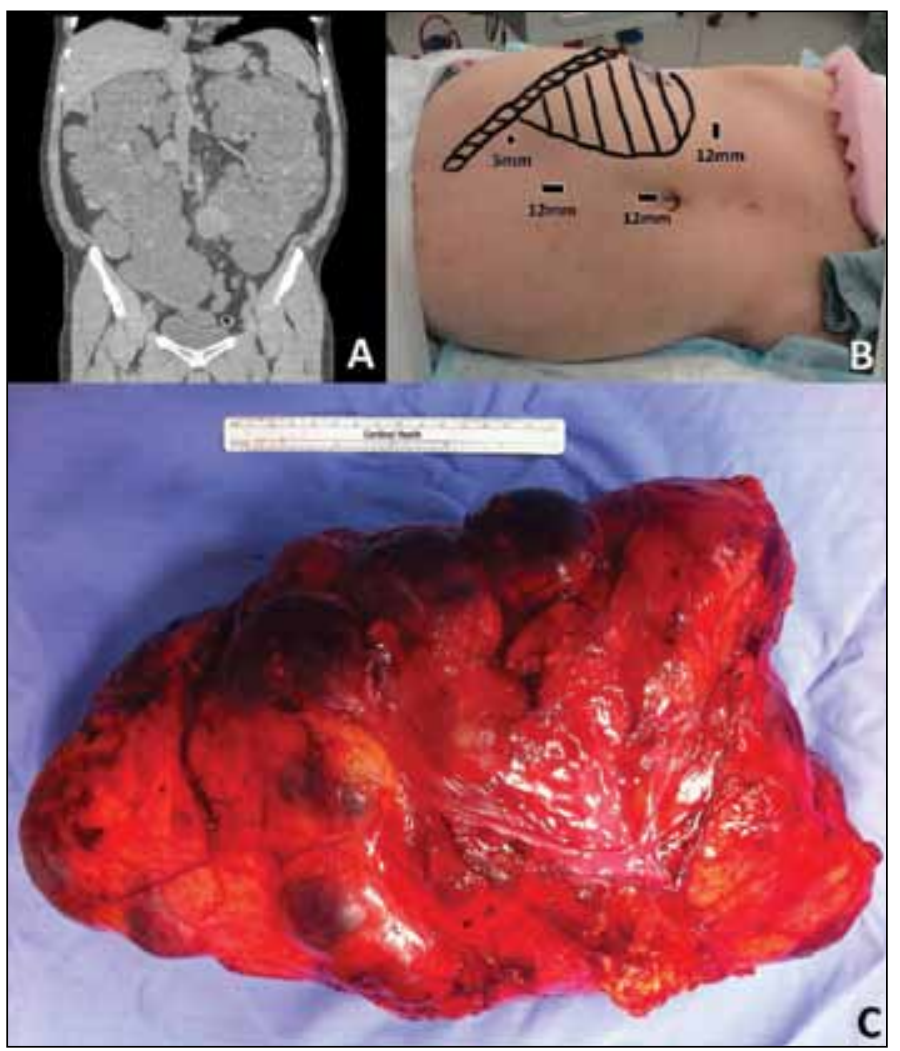

Fig. 1. A: Coronal computed tomography view of 49 -year-old male before laparoscopic nephrectomy; B: Position of the patient and port placement; C: Specimen of massively enlarged polycystic kidney.
The surgeon and the assistant stand anterior to the patient. Video monitors are located at the head of the operating table on both sides. A $15-\mathrm{mm} \mathrm{HgCO}_{2}$ pneumoperitoneum is established with a Veress cannula that is placed at the apex of the umbilicus or alternatively by using a 12-mm Optiview (Ethicon Endo-Surgery, Inc., Cincinnati, OH) direct laparoscopic access technique. Two additional 12-mm ports and one 5-mm port are placed in a gentle arc-like shape under direct vision (Fig. 1, part B). We used an additional $5-\mathrm{mm}$ port in the subxiphoid region to retract the liver for the right-sided nephrectomy. A 0-degree-viewing 10-mm laparoscope is inserted initially for ports insertion and then exchanged with a 30-degree-viewing 10-mm laparoscope that is used throughout the procedure. On the right side, the colon is mobilized toward the midline, and the duodenum is kocherized to expose the kidney. On the left, the descending colon and spleen are mobilized medially to optimize exposure of the entire kidney.

It is important to be aware of the location of the mesocolon, spleen, pancreas and gallbladder, because they frequently adhere to the cystic kidney. Previously, care was taken to minimize the cyst puncture which may lead to spillage and possible peritonitis. Currently, judicious puncture and suction of large clear cysts is used to facilitate dissection. Whenever required for massively enlarged kidneys (Fig. 1, part C), we used the umbilical port as the camera port and the rest as working ports for dissection of mid and lower pole of the kidney and the midline epigastric port as the camera port and others as working ports for dissection of the upper and mid pole. Interchanging camera ports helped visualize the upper and lower poles of massive polycystic kidneys. To help identify the right renal vein, the lateral border of the inferior vena cava (on the right) can be used as a guide. The ureter identified at the pelvic brim (right or left) can also be used as a landmark and followed proximally to the hilum. If necessary, extra 5-mm ports can be inserted to negotiate the large polycystic kidney. To retract portions of the large polycystic renal mass and facilitate dissection, we find the Jarit Padron Endoscopic Exposing Retractor (PEER) (J. Jamner Surgical Instruments, Inc, Hawthorne, NY) useful (Fig. 2).

Both the artery and ureter were divided after being controlled by multiple titanium clips. The renal vein was divided using the EndoGIA autosuture device. The adrenal gland was preserved whenever possible. The kidney was extracted intact through an infraumbilical midline, Pfannenstiel or extended port-site incision according to surgeon's preference. Often, the kidney was too large to fit into the endocatch bag and was removed without it. The extraction incision was closed, and pneumoperitoneum recreated to inspect for hemostasis. We irrigated the abdomen with 2 litres of saline to wash any residual cyst fluid. The abdomen was deflated, and the 12-mm trocar incisions were closed under direct vision with absorbable sutures, using the Carter-Thomason device. The 5-mm ports were closed only at the skin level. 


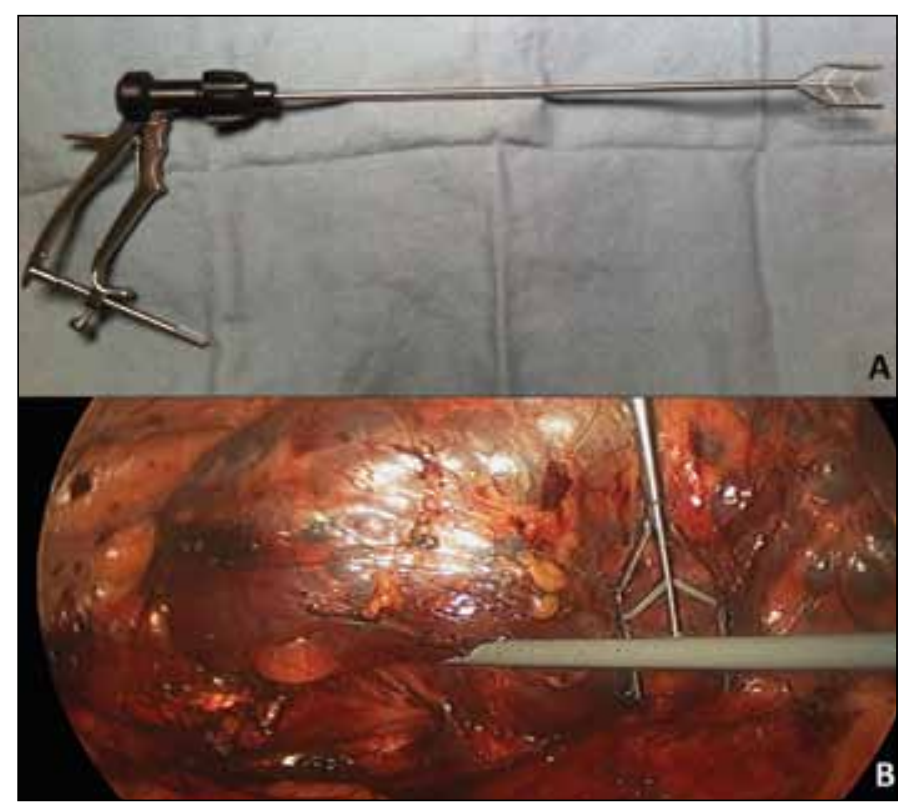

Fig. 2. A: Jarit Padron Endoscopic Exposing Retractor (PEER) (J. Jamner Surgical Instruments, Inc, Hawthorne, NY); B: The retractor being used during the laparoscopic nephrectomy for autosomal dominant polycystic kidney disease.

\section{Results}

During the 7-year study period, 32 patients (male [M] 21, female [F] 11) underwent laparoscopic nephrectomies for 39 renal units. Seven patients $(M=6, F=1)$ underwent staged bilateral nephrectomy and 25 patients $(M=15, F=10)$ underwent unilateral laparoscopic nephrectomy. Fourteen were left sided and 25 were right sided. Surgical indications were varied: to create space for future renal transplant in 21 , to alleviate pain in 16 , to prevent recurrent urosepsis in 2, to prevent recurrent bleeding which would require transfusions in 2 and to remove a renal tumour in 1 kidney. For patients had surgery for more than one reason. Preoperative parameters are outlined in Table 1. Fourteen (36\%) patients had undergone prior abdominal surgeries, 27 (69\%) patients were receiving hemodialysis and $3(8 \%)$ patients were on

\begin{tabular}{lc}
\hline Table 1. Preoperative parameters \\
\hline No. cases $(\mathrm{n})$ & 39 \\
No. patients $(\mathrm{n})$ & 32 \\
Sex (Male:Female) & $21: 11$ \\
Mean age (years) & 52.2 (range: $29-72$ ) \\
Median ASA class & 3 \\
Mean BMI (kg/m2) & 26.9 (range: 21.6-34.0) \\
Preoperative dialysis (n) & 30 (HD 27, PD 3) \\
Operative side (Left:Right) & $14: 25$ \\
Mean preoperative creatinine ( $\mu$ mol/L) & 514 (range: $84-923$ ) \\
Mean preoperative hemoglobin (g/L) & 131.6 (range: 107-171) \\
Previous abdominal surgery (n) & 14 \\
\hline ASA: American Society of Anesthesiologists classification; BMl: body mass index, HD: \\
hemodialysis, PD: peritoneal dialysis.
\end{tabular}

peritoneal dialysis at the time of nephrectomy. Seven (18\%) patients had undergone nephrectomy after the renal transplant. Out of 32 patients, $26(81 \%)$ patients received a kidney transplant, either prior to nephrectomy (7 patients) or after the nephrectomy (19 patients). Six patients were still on the wait-list for the transplant. No patient underwent angioembolization preoperatively. Table 2 outlines the perioperative data. The mean surgical duration was 185 minutes (range: 113-287); and the estimated blood loss was $94 \mathrm{~mL}$ (range: 10-350). Surgeons required 5 laparoscopic ports for operation in 6 procedures $(15 \%), 4$ ports in $9(23 \%)$ procedures and 3 ports in $24(62 \%)$ procedures. Gelport was required in 1 patient, in addition to 3 laparoscopic ports, due to difficulty. No patient required open conversion. The specimen was extracted through a Pfannenstiel incision in 6 procedures, a midline incision in 26 procedures and incision extending one of the port-site was used in 7 procedures. The length of incision for extraction of intact specimen ranged from 6 to $13 \mathrm{~cm}$ with a mean of $9.2 \mathrm{~cm}$. The mean weight of final specimen was $1515 \mathrm{~g}$ (range: 412-4590); and mean size was $24.2 \mathrm{~cm}$ (range 15-38). Histological diagnosis of ADPKD was confirmed in all the specimens; 1 patient also had $3-\mathrm{cm}$ papillary type 1 renal cell carcinoma (RCC). The patient with papillary type 1 RCC was recurrence/metastasis free at the 2-year follow-up. The mean length of stay was 4.5 days (range: $3-8$ ). Unassisted ambulation started on an average in 2 days (range: 1-7) and full diet in 2 days (range: 2-7). Postoperatively, the levels of hemoglobin were at a mean of $117.6 \mathrm{~g} / \mathrm{L}$ (range: 95-147).

There were 4 complications (Table 3 ). One Grade 2 complication required blood transfusions for postoperative bleeding from contralateral kidney. Two Grade 3b complications came from a gall bladder perforation requiring cholecystectomy and a umbilical port-site hernia requiring repair later. The single Grade 4a-d complication was postoperative sepsis and myocardial infarction requiring prolonged

\begin{tabular}{|c|c|c|}
\hline \multicolumn{2}{|c|}{ Mean duration of procedure (min) } & $185(113-287)$ \\
\hline \multicolumn{2}{|c|}{ Mean estimated blood loss (mL) } & $94(10-350)$ \\
\hline \multicolumn{2}{|c|}{ Mean size of extraction incision $(\mathrm{cm})$} & $9.2(6-13)$ \\
\hline \multirow[t]{3}{*}{ Extraction type $(n)$} & Pfannensteil & 6 \\
\hline & Midline infraumbilical & 26 \\
\hline & Port-site extended & 7 \\
\hline \multicolumn{2}{|c|}{ Mean specimen weight (g) } & $1515(412-4590)$ \\
\hline \multicolumn{2}{|c|}{ Mean specimen size $(\mathrm{cm})$} & $24.2(15-38)$ \\
\hline \multicolumn{2}{|c|}{ Mean postoperative hemoglobin ( $\mathrm{g} / \mathrm{L}$ ) } & $117.6(95-147)$ \\
\hline \multicolumn{2}{|c|}{ Mean length of stay (days) } & $4.5(3-8)$ \\
\hline \multicolumn{2}{|c|}{ Mean unassisted ambulation (days) } & $2(1-7)$ \\
\hline \multicolumn{2}{|c|}{ Mean first full diet (days) } & $2(2-7)$ \\
\hline \multirow[t]{2}{*}{ Histopathology (n) } & Benign & 38 \\
\hline & Malignant & $\begin{array}{l}1 \text { (type } 1 \text { papillary } \\
\text { renal cell carcinoma) }\end{array}$ \\
\hline
\end{tabular}




\begin{tabular}{ll}
\hline $\begin{array}{l}\text { Table 3. Complications according to Modified Clavien } \\
\text { System }\end{array}$ \\
\hline Grade 1 & 0 \\
Grade 2 & 1 \\
Grade 3a & 0 \\
Grade 3b & 2 \\
Grade 4a-d* & 1 \\
Grade 4b & 0 \\
Grade 5 & 0 \\
\hline *Suffix dindicates need for follow-up to fully evaluate the complication. \\
\hline
\end{tabular}

intensive cardiac unit stay. This patient was the first case in our series and had rupture of cysts intraoperatively to make space to help dissection. Retrospectively, the irritation by complex cyst fluid might have led to peritonitis and sepsis. We currently selectively puncture and aspirate the larger clear cysts and do not puncture any cyst that may contain debris or complexity. There were no deaths.

\section{Discussion}

Most patients with ADPKD can be asymptomatic, but a small percentage may get massively enlarged kidneys with resultant problems. Symptoms related to enlarged kidneys may be in the form of pain, abdominal fullness, gastro-esophageal reflux and infection. Most of these symptoms are generally managed using medical treatment; surgery is offered to patients failing conservative methods. Nephrectomy is indicated for patients with large kidneys causing pressure symptoms, pain, infection, bleeding, hypertension, erythrocytosis and suspicion of malignancy; nephrectomy is also indicated in these patients to create space for renal allograft. ${ }^{11}$ As reported previously, open nephrectomy for enlarged polycystic kidneys in patients with ADPKD is associated with significant complication rates. ${ }^{3}$ In azotemic patients especially, larger abdominal incision adds to existing comorbidities and prolongs the convalescence period. Because of high morbidity and mortality and lack of complications in native polycystic kidneys after the transplants, in the Ho-Hsieh and colleagues' study, open nephrectomy for polycystic kidneys was performed less frequently. ${ }^{12}$

Since the description of laparoscopic nephrectomy in ADPKD by Elashry and colleagues, many centres have described their experience with pure laparoscopic or hand-assisted laparoscopic nephrectomy for ADPKD. ${ }^{7-9,13-16}$ Binsaleh and colleagues compared laparoscopic and open approaches and concluded that laparoscopic nephrectomy is a feasible and safe alternative to open nephrectomy in patients with ADPKD. They also showed laparoscopy resulted in shorter incision, less pain, shorter length of stay and excellent cosmesis as compared to open nephrectomy. ${ }^{9}$ Similarly, Dunn and colleagues, in their series of 11 patients with ADPKD who underwent laparoscopic nephrectomy, reported minimal intraoperative estimated blood loss, less pain, shorter length of hospital stay and rapid recovery. ${ }^{13}$

As shown by Bendavid and colleagues, in 22 patients who underwent laparoscopic nephrectomies for ADPKD with median kidney size of $22 \mathrm{~cm}$ (range: 8-50), the reported intraoperative and postoperative complication rate was $18 \%$ and $32 \%$, respectively. They reported median surgical time as 255 minutes (range: 95-415) and conversion to open rate of $18 \%$. They concluded that laparoscopic nephrectomy is a safe procedure, offering short length of hospital stay, but with comparatively high morbidity and conversion rate. ${ }^{14}$ Lipke and colleagues successfully reported bilateral hand-assisted laparoscopic nephrectomy for 18 patients, 4 of which were converted because of the large size of the polycystic kidneys. They used 8-cm periumbilical incision for the hand-assisted LapDisc (Ethicon Endo-Surgery, Inc., Cincinnati, $\mathrm{OH}$ ) device and for the removal of the specimen after significant decortication. They concluded that patients with massively enlarged polycystic kidneys (>3500 cc) may benefit from open nephrectomy. ${ }^{?}$

In their retrospective review of 10 patients undergoing bilateral hand-assisted laparoscopic nephrectomy, Whitten and colleagues successfully reported the use of vacuum curettage to reduce kidney size and facilitate the removal through a periumbilical hand port incision. ${ }^{15}$ We preferred not to morcellate and decided to remove the specimens intact in our series. The spillage of cyst contents and fluid in the peritoneal cavity has been associated with pain and peritonitis with subsequent ileus. This happened in one of our patients who had cysts ruptured intraoperatively with spillage of contents and developed peritonitis and sepsis requiring intensive medical therapy subsequently. However, any other source of infection and sepsis apart from cyst fluid could not be ruled out in the same patient; laparoscopic cyst de-roofing is a commonly performed procedure in which cyst fluid spillage occurs. We feel that careful aspiration of the clear cyst fluid by Veress needle might help minimize the risk of peritoneal irritation or infection, but larger controlled studies are required. Morcellation of massively enlarged kidney would also add significant time (30-60 minutes) to the operative procedure. Also, there is a rare but plausible chance of malignancy in the kidney. ${ }^{13}$ One of our other patients was diagnosed with $3-\mathrm{cm}$ papillary RCC on histopathological examination and was recurrence/ metastasis free at the 2-year follow-up. Although, sometimes it is difficult to entrap the specimen completely due to its size, we still recommend bagging the specimen whenever feasible, as it can facilitate extraction through a smaller incision and prevent undue cyst fluid and rare tumour spillage. The mean length of hospital stay in our case series (4.5 days) was higher than in many other series, ${ }^{7,8,14}$ but less than in the series by Desai and colleagues (4.86 days). ${ }^{17}$ This might have 
been due to the fact that our mean ASA (American Society of Anesthesiologists) scores, size and weight of the kidneys were higher than in other series.

The conversion to open surgery in various series is $9 \%$ to $22 \%{ }^{7,14,17,18}$ Considering the large size of the kidneys, the availability of space is limited and incidental injury to other adjacent organs should be kept in mind. In a report by Ivey and colleagues, ${ }^{18}$ there was significantly higher open conversion rate $(40 \%$ vs. $8.2 \%, p<0.001)$, higher mean estimated blood loss (400 vs. $100 \mathrm{~mL}, p<0.001)$, longer operative time (5.6 vs. $3.25 \mathrm{~h}, p=0.003$ ) and longer stay in hospital (4.0 vs. $3.0 \mathrm{~d}, p=0.046$ ) for laparoscopic nephrectomies involving specimen weight $>1500 \mathrm{~g}$. In the present series, no open conversion was required. This is probably due primary surgeon's expertise and comfort level with advanced laparoscopic procedures. We advocate the use of either Optiview trocar or Hasson cannula for direct laparoscopic access as Veress needle may injure intra-abdominal organs due to space limits.

Laparoscopic nephrectomy for ADPKD differs from standard nephrectomy for other renal conditions because of space constraints and the large kidneys. The dissection of adrenal and postero-lateral portions of the kidney is especially challenging and may require additional port placement or hand-assisted device. The use of extra $12-\mathrm{mm}$ or $5-\mathrm{mm}$ ports is encouraged whenever required. We found Jarit Padron Endoscopic Exposing Retractor (PEER) (Fig. 2) especially useful for this purpose. We successfully used $12-\mathrm{mm}$ umbilical and epigastric ports as camera ports to dissect the lower and upper pole, respectively.

This study has some limitations. It is retrospective and lacks a comparative open group, but it may not be appropriate to offer open surgery in today's era of laparoscopy.

\section{Conclusion}

Our updated technique of transperitoneal laparoscopic nephrectomy for massively enlarged polycystic kidneys in patients with ADPKD is safe and offers all the advantages of minimal access surgery, such as reduced estimated blood loss, smaller incision, excellent cosmesis and faster recovery. As laparoscopic nephrectomy in ADPKD has a significant learning curve, we encourage surgeons to gain significant experience in advanced laparoscopy before trying this procedure so that complications and conversions can be minimized.

Competing interests: Dr. Bansal declares no competing financial or personal interests. Dr. Kapoor is a member of the Speakers bureau for, and has received grants and honoraria from, Pfizer Oncology, GSK Oncology, Novartis Oncology and Amgen. He has also participated in clinical trials within the past 2 years with NCIC, Pfizer, GSK, Novartis and Amgen.
This paper has been peer-reviewed.

\section{References}

1. Igarashi P, Somolo S. Genetics and pathogenesis of polycystic kidney disease. J Am Soc Nephrol 2002;13:2384-98. http://dx.doi.org/10.1097/01.ASN.0000028643.17901.42

2. Torres VE, Harris PC, Pirson Y. Autosomal dominant polycystic kidney disease. Lancet 2007;369:1287301. http://dx.doi.org/10.1016/S0140-6736(07)60601-1

3. Bennett AH, Stewart W, Lazarus JM. Bilateral nephrectomy in patients with polycystic renal disease. Surg Gynecol Obstet 1973;137:819-20.

4. Knispel HH, Klan R, Offermann G, et al. Transplantation in autosomal dominant polycystic kidney disease without nephrectomy. Urol Int 1996;56:75-8. http://dx.doi.org/10.1159/000282815

5. Sulikowski T, Tejchman K, Zietek Z, et al. Experience with autosomal dominant polycystic kidney disease in patients before and after renal transplantation: A 7-year observation. Transplant Proc 2009;41:177-80. http://dx.doi.org/10.1016/i.transproceed.2008.10.034

6. Elashry OM, Nakada SY, Wolf JS Jr, et al. Laparoscopy for adult polycystic kidney disease: a promising alternative. Am J Kidney Dis 1996;27:224-33. http://dx.doi.org/10.1016/S0272-6386(96)90545-4

7. Lipke MC, Bargman V, Milgrom M, et al. Limitations of laparoscopy for bilateral nephrectomy for autosomal dominant polycystic kidney disease. J Urol 2007;177:627-31. http://dx.doi.org/10.1016/i. juro.2006.09.026

8. Binsaleh S, Al-Enezi A, Dong J, et al. Laparoscopic nephrectomy with intact specimen extraction for polycystic kidney disease. J Endourol 2008;22:675-9. http://dx.doi.org/10.1089/end.2007.0147

9. Binsaleh S, Luke PP, Nguan C, et al. Comparison of laparoscopic and open nephrectomy for adult polycystic kidney disease: Operative challenges and technique. Can I Urol 2006;13:3340-5.

10. Dindo D, Demartines N, Clavien PA. Classification of Surgical Complications. A new proposal with evaluation in a cohort of 6336 patients and results of a survey. Ann Surg 2004;240:205-13. http://dx.doi. org/10.1097/01.sla.0000133083.54934.ae

11. Hadimeri $H$, Norden $G$, Friman $S$, et al. Autosomal dominant polycystic kidney in a kidney transplant population. Nephrol Dialysis Transplant 1997;12:1431-6. http://dx.doi.org/10.1093/ndt/12.7.1431

12. Ho-Hsieh H, Novick AC, Steinmuller D, et al. Renal transplantation for end-stage polycystic kidney disease. Urology 1987;30:322-6. http://dx.doi.org/10.1016/0090-4295(87)90293-7

13. Dunn MD, Portis AJ, Elbahnasy AM, et al. Laparoscopic nephrectomy in patients with end-stage renal disease and autosomal dominant polycystic kidney disease. Am J Kidney Dis 2000;35:720-5. http:// dx.doi.org/10.1016/50272-6386(00)70021-7

14. Bendavid Y, Moloo H, Klein L, et al. Laparoscopic nephrectomy for autosomal dominant polycystic kidney disease. Surg Endosc 2004;18:751-4. http://dx.doi.org/10.1007/s00464-003-9172-z

15. Whitten MG, Van der Werf $W$, Belnap L. A novel approach to bilateral hand-assisted laparoscopic nephrectomy for autosomal dominant polycystic kidney disease. Surg Endosc 2006;20:679-84. http://dx.doi. org/10.1007/s00464-005-0229-z

16. Lee DI, Clayman RV. Hand-assisted laparoscopic nephrectomy in autosomal dominant polycystic kidney disease. J Endourol 2004;18:379-82. http://dx.doi.org/10.1089/089277904323056942

17. Desai MR, Nandkishore SK, Ganpule A, et al. Pretransplant laparoscopic nephrectomy in adult polycystic kidney disease: a single centre experience. BJU Int 2007;101:94-7.

18. Ivey BS, Lucas SM, Meyer CA, et al. Conversions in laparoscopic renal surgery: Causes and outcomes. J Endourol 2011;25:1167-73. http://dx.doi.org/10.1089/end.2010.0725

Correspondence: Dr. Anil Kapoor, McMaster Institute of Urology, St. Joseph's Healthcare, Hamilton, ON; akapoor@mcmaster.ca 